\title{
Meson correlators in a finite volume near the chiral limit
}

\section{Hidenori Fukaya}

Yukawa Institute for Theoretical Physics, Kyoto University, Kyoto 606-8052, Japan.

\section{Shoji Hashimoto}

High Energy Accelerator Research Organization (KEK), Tsukuba 305-0801, Japan.

School of High Energy Accelerator Science, The Graduate University for Advanced Studies

(Sokendai), Tsukuba 305-0801, Japan.

\section{Kenji Ogawa ${ }^{* \dagger}$}

School of High Energy Accelerator Science, The Graduate University for Advanced Studies (Sokendai), Tsukuba 305-0801, Japan.

E-mail: ggawak@post.kek.jp

\begin{abstract}
We report on the results of our calculation of meson correlators in a finite volume. The calculation is carried out in the quenched approximation near the chiral limit (down to $m_{q}=2.6 \mathrm{MeV}$ ) using the overlap fermion. For these small quark masses, the scalar and pseudo-scalar correlators are well approximated with a few hundred eigenmodes. The results for both connected and disconnected correlators are compared with the theoretical predictions of quenched chiral perturbation theory.
\end{abstract}

XXIIIrd International Symposium on Lattice Field Theory

25-30 July 2005

Trinity College, Dublin, Ireland

\footnotetext{
*Speaker.

${ }^{\dagger}$ KEK-CP-168
} 


\section{Introduction}

In a finite volume the meson correlators are largely distorted. Under an extreme condition such as the $\varepsilon$-regime [1], the correlators do not even show the familiar exponential fall-off as a function of $t$. It is, however, still possible to analytically describe the system using the chiral perturbation theory (ChPT), as long as the pion mass is small enough. One-loop calculations in the quenched approximation are available for both connected and disconnected correlators [2, 3]. Since they are expressed in terms of the usual low energy constants (LECs) in ChPT, the lattice QCD may be used to determine their values through the calculation of meson correlators in the $\varepsilon$-regime.

In this work we calculate the quenched meson correlators near the chiral limit. We employ the overlap-Dirac operator and calculate low-lying eigenvalues and eigenvectors in order to investigate the low-lying mode saturation of the meson correlators. For the scalar and pseudo-scalar correlators the saturation is well satisfied, and we can obtain them very close to the chiral limit without extra computing cost. We present our result for the pion decay constant $F_{\pi}$ and chiral condensate $\Sigma$. A full detail of this work is given in [4] (see also [5]). Other works in this direction include [6, , $, 8,9]$.

\section{Simulation methods}

We work on a $10^{3} \times 20$ lattice at $\beta=5.85$ in the quenched approximation. The physical lattice spacing corresponds to $0.123 \mathrm{fm}$. We use the overlap-Dirac operator

$$
D_{m}=\left(1-\frac{\bar{a} m}{2}\right) D+m, D=\frac{1}{\bar{a}}\left(1+\gamma_{5} \operatorname{sgn}\left(a H_{W}\right)\right), H_{W}=\gamma_{5}\left(D_{W}-\frac{1}{\bar{a}}\right),
$$

with $\bar{a} \equiv a /(1+s)$. The parameter $s$ is set to 0.6 . In the computation of the sign function (2.1), we treat 60 lowest eigenmodes of $H_{W}$ exactly and approximate the rest by the Chebyshev polynomial (degree 100-200). The topological sectors are identified unambiguously by counting the number of zero mode of $D$. We analyzed 20, 45, 44 and 24 gauge configurations for topological charge $|Q|=$ $0,1,2$ and 3, respectively. The 200 lowest eigenvalues and associated eigenvectors of the overlapDirac operator $D$ are calculated using the implicitly restarted Arnoldi method (implemented in the ARPACK numerical package).

Near the massless limit, the quark propagator is expected to be well approximated by these low-lying eigenvectors.

$$
D_{m}^{-1}(x, y) \sim \sum_{i=1}^{200+|Q|} \frac{1}{(1-\bar{a} m / 2) \lambda_{i}+m} v_{i}(x) v_{i}^{\dagger}(y)
$$

where $\lambda_{i}$ and $v_{i}(x)$ are the $i$-th eigenvalue and eigenvector, respectively. In Figure 1 1 we compare the pseudo-scalar (left panel) and axial-vector (right panel) correlators constructed from the approximated quark propagator (2.2) with those from the exact propagator (obtained using the conventional CG inverter). At a small quark mass $a m=0.008$, we find a good agreement of the approximate correlator with the exact one for the pseudo-scalar correlator. Such saturation becomes even better for smaller quark masses and higher topological sectors. The saturation is, on the other hand, much worse for the axial-vector correlator. Therefore, in the following analysis the axial-vector correlators are calculated with the exact method, while the low-mode approximation is used for quantities 

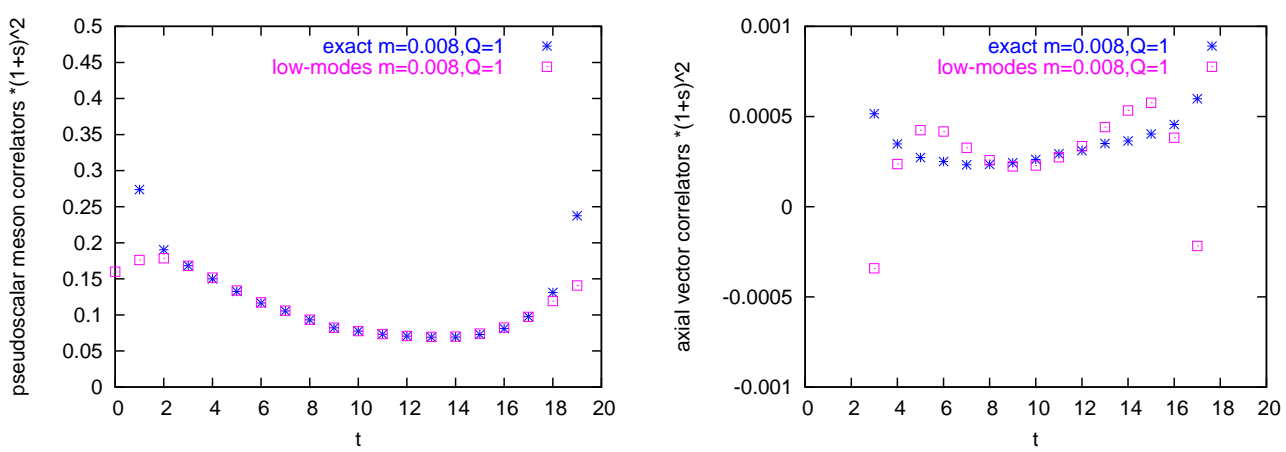

Figure 1: Comparison of the pseudo-scalar (left) and axial-vector (right) correlators evaluated with the low-mode approximation (stars) with the exactly solved correlators (open squares).

for which the saturation is well satisfied. One advantage of the low-mode approximation (2.2) is that one can reduce the statistical noise drastically using the low-mode averaging technique [7] 10] without much extra computing cost.

\section{Axial-vector current correlator}

For the local axial-vector current $A_{\mu}^{a}=\bar{\psi} \gamma_{5} \gamma_{\mu}\left(\tau^{a} / 2\right) \psi$ we calculate the renormalization factor $Z_{A}$ non-perturbatively using the Ward-Takahashi identity. $>$ From the ratio of $\left\langle\bar{\nabla}_{0} A_{0}^{3}(t) P^{3}(0)\right\rangle$ and $\left\langle P^{3}(t) P^{3}(0)\right\rangle$ correlators, $Z_{A}$ is obtained as a linear slope as a function of $m$. Our result is $Z_{A}=$ $1.439(15)$.

In Figure 2 we show the results for the axial-vector correlators for topological charges $|Q|=$ 0,1 and 2. The expected form from the quenched ChPT (QChPT) is written as

$$
Z_{A}^{2}\left\langle A_{0}^{3}(t) A_{0}^{3 \dagger}\right\rangle=\frac{F_{\pi}^{2}}{T}+2 m \Sigma_{|Q|}(\mu) T h_{1}(t / T) .
$$

where $\Sigma_{Q}(\mu)$ is the scalar condensate at a given topological charge $Q$ and $\mu=m \Sigma V: \Sigma_{Q}(\mu) / \Sigma=$ $\mu\left(I_{Q}(\mu) K_{Q}(\mu)+I_{Q+1}(\mu) K_{Q-1}(\mu)\right)+Q / \mu .\left(I_{Q}(\mu)\right.$ and $K_{Q}(\mu)$ denote the modified Bessel functions.) The $t$ dependence is simply given by a quadratic function $h_{1}(x)=1 / 2\left((x-1 / 2)^{2}-1 / 12\right)$. $>$ From this expression, $F_{\pi}$ is determined through the constant term, while the second term has dependence on $|Q|$ and $\mu$ as well as $t / T$. >From a simultaneous fit for the data at $|Q|=0$ and 1 with several values of $m$ and $t / T$, we obtain $F_{\pi}=98.3(8.3) \mathrm{MeV}$ and $\Sigma^{1 / 3}=259(50) \mathrm{MeV}$. The formula (3.1) explains the data very well at $|Q|=0$ and 1 , but the same set of parameters does not describe the data at higher topological charge $(|Q|=2$, shown in the right panel of Figure 2). This is not inconsistent with the theoretical expectation, because the derivation of $(3.1)$ assumes a condition $|Q| \ll\left\langle Q^{2}\right\rangle$ and for our small lattice $\left\langle Q^{2}\right\rangle=4.34(22)$. For more detail, see [4].

\section{Connected scalar and pseudo-scalar correlators}

The connected scalar and pseudo-scalar correlators are obtained using the low-mode approximation. Since we can average the source point over the space-time lattice, the signal is very clean as shown in Figure . 


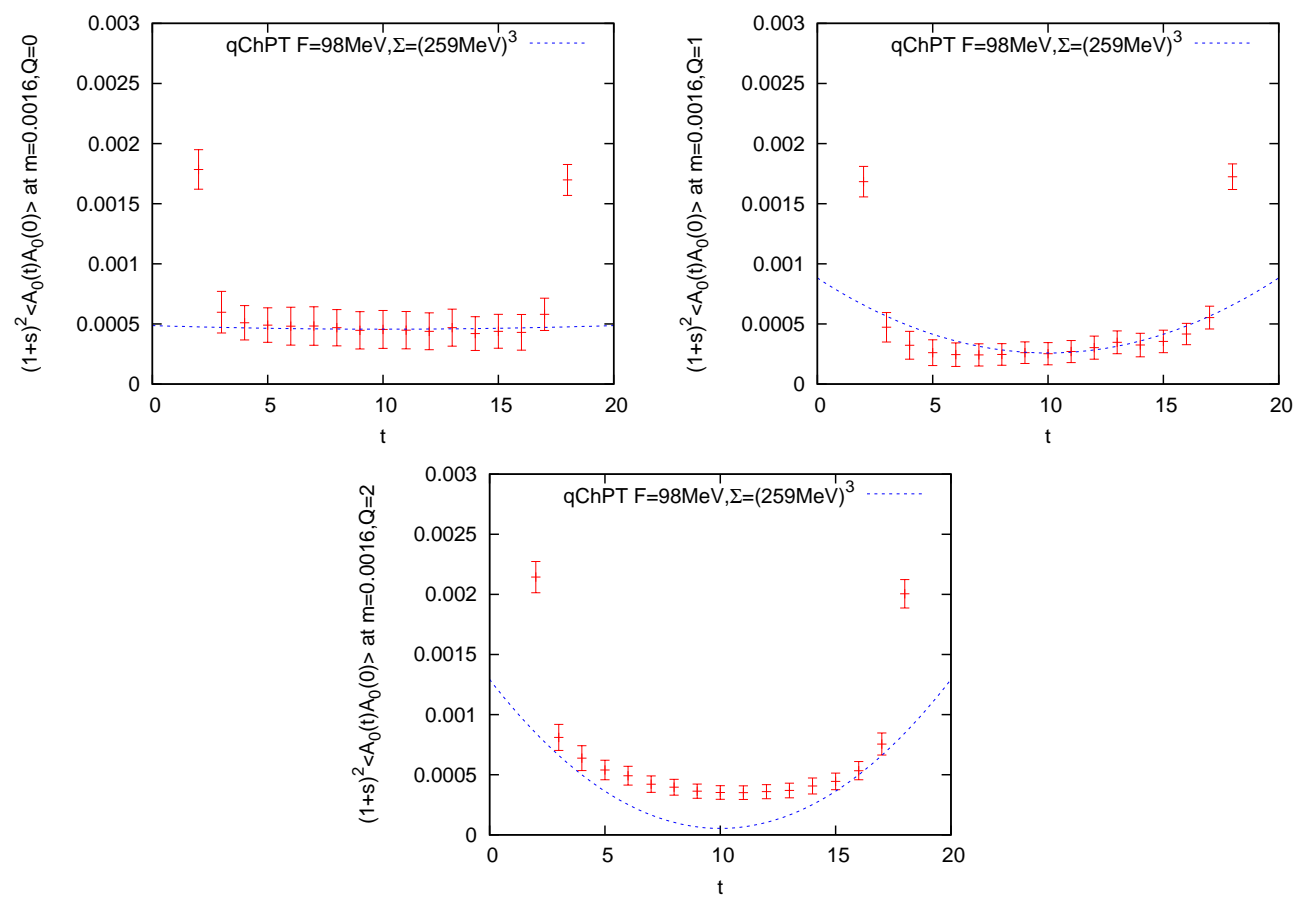

Figure 2: Axial-vector current correlator at $Q=0$ (top left), $|Q|=1$ (top right) and $|Q|=2$ (bottom). The lines show the fit with the function (3.1) for $|Q|=0$ and 1 .

The fitting with the QChPT expectation is more involved than the case of the axial-vector current correlator, because the parameters $m_{0}^{2}$ and $\alpha$ appear to describe the quenched artifacts. To avoid a four-parameter fit with $\left(F_{\pi}, \Sigma, m_{0}^{2}, \alpha\right)$, we input $F_{\pi}$ from the result of the axial-vector current correlator and use the relation $\left\langle Q^{2}\right\rangle / V=m_{0}^{2} F_{\pi} / 2 N_{c}$ to determine $m_{0}^{2}$.

The data shown in Figure 3 are well described by the QChPT expectation for $|Q|=0$ and 1 , as in the axial-vector current correlator. We obtain $\Sigma^{1 / 3}=257(14)(00) \mathrm{MeV}$ and $\alpha=-4.5(1.2)(0.2)$, where the first error is statistical while the second represents the uncertainty of input parameters.

Our result for $\alpha$ is rather large and negative, which contradict with the small $\alpha$ observed in many previous lattice calculations (for example, see [11]). We also attempted a fit with $F_{\pi}$ and $\Sigma$ as free parameters, while $\alpha$ is set equal to zero. The result is $F_{\pi}=136.9(5.3)(0.9) \mathrm{MeV}$ and $\Sigma^{1 / 3}=$ $250(13)(00) \mathrm{MeV} ; F_{\pi}$ is not consistent with the analysis of the axial-vector current correlators.

\section{Disconnected pseudo-scalar correlator}

We also study the disconnected pseudo-scalar correlator using the low-mode approximation. For this quantity we did not check the consistency with the exact solution because it is too costly. Instead, we looked at the saturation as the number of eigenmodes included in the approximation. Figure $\bigoplus$ shows that the disconnected diagram is well saturated with the number of eigenvalues greater than 10 .

Data of the disconnected pseudo-scalar correlators are shown in Figure F. As in the connected correlator, we fit to the QChPT formula with $\Sigma$ and $\alpha$ as free parameters. The result for $|Q|=0$ and 

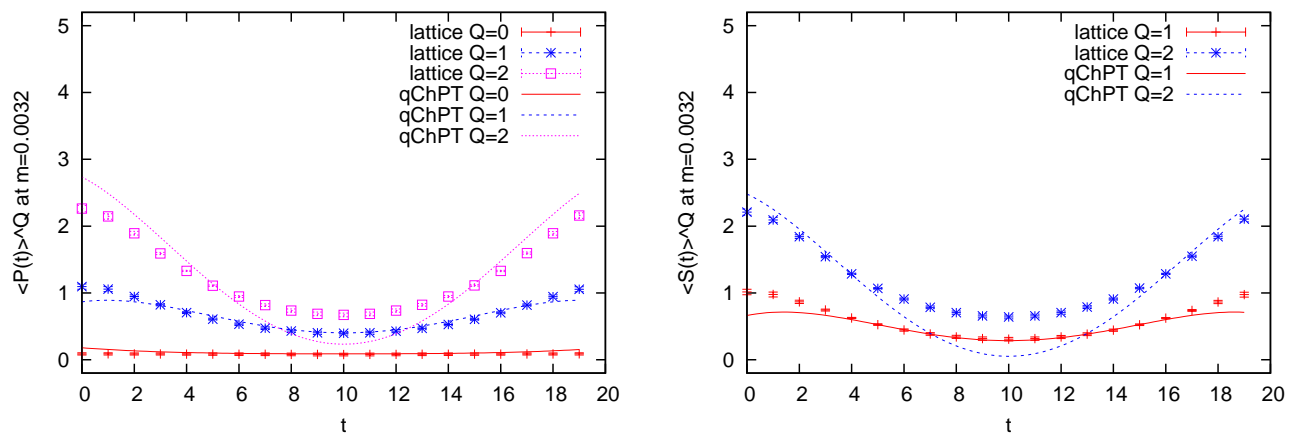

Figure 3: Connected pseudo-scalar (left) and scalar (right) correlators. The curves show the fitting with the analytically predicted form.

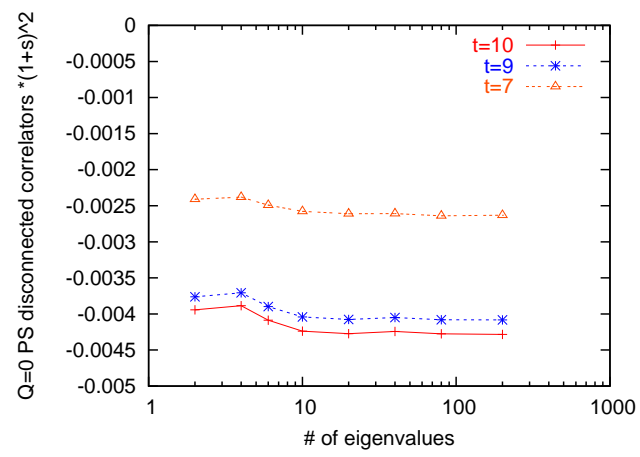

Figure 4: Saturation of the disconnected correlator at $t=7,9$ and 10 as a function of the number of eigenvalues in the lowmode approximation.

1 data is $\Sigma^{1 / 3}=227(32)(00) \mathrm{MeV}$ and $\alpha=-3.5(1.2)(0.3)$, which is consistent with the connected correlators. The analysis with $\alpha=0$ yields $F_{\pi}=125.7(5.6)(0.9) \mathrm{MeV}$ and $\Sigma^{1 / 3}=223(29)(00) \mathrm{MeV}$.

\section{Summary}

We performed a quenched simulation in the $\varepsilon$-regime at small quark masses $m=2.6-13 \mathrm{MeV}$. In this small quark mass region, the connected scalar and pseudo-scaler correlators are approximated by the low-lying modes of the Dirac operator to $1 \%$ accuracy or even better. In the small topological sector, the results are well described by the one-loop QChPT formula; by fitting we can extract $F_{\pi}$ and $\Sigma$ as well as $m_{0}^{2}$ and $\alpha$.

We also find problems: Data at higher topological sectors are not consistent with the QChPT expectation. Even for the small $|Q|$ sector, the large negative value of $\alpha$ may suggest a breakdown of the theoretical expression. We suspect that these problems arises from the small physical volume of our lattice for which the condition $|Q| \ll\left\langle Q^{2}\right\rangle$ is not well satisfied except for $Q=0$.

\section{References}

[1] J. Gasser and H. Leutwyler, Thermodynamics Of Chiral Symmetry Phys. Lett. B 188, 477 (1987). 

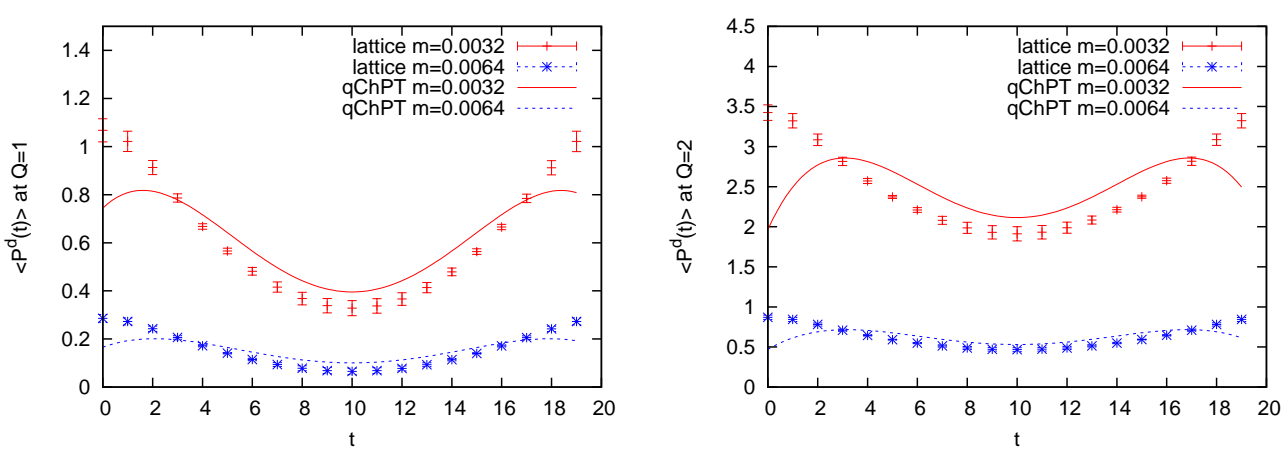

Figure 5: Disconnected pseudo-scalar correlators for $|Q|=1$ (left) and $|Q|=2$ (right). The lines are the fitting function with the parameters which was obtained by calculating axial-vector current correlators and connected pseudo-scalar and scalar correlators.

[2] P. H. Damgaard, M. C. Diamantini, P. Hernandez and K. Jansen, Finite-size scaling of meson propagators Nucl. Phys. B 629, 445 (2002) [arXiv:hep-lat/0112016].

[3] P. H. Damgaard, P. Hernandez, K. Jansen, M. Laine and L. Lellouch, Finite-size scaling of vector and axial current correlators Nucl. Phys. B 656, 226 (2003) [arXiv:hep-lat/0211020].

[4] H. Fukaya, S. Hashimoto and K. Ogawa, Low-lying mode contribution to the quenched meson correlators in the epsilon-regime arXiv:hep-lat/0504018.

[5] K. Ogawa and S. Hashimoto, Effect of low-lying fermion modes in the epsilon-regime of QCD arXiv:hep-lat/0505017.

[6] W. Bietenholz, T. Chiarappa, K. Jansen, K. I. Nagai and S. Shcheredin, Axial correlation functions in the epsilon-regime: A numerical study with overlap fermions JHEP 0402, 023 (2004) [arXiv:hep-lat/0311012].

[7] L. Giusti, P. Hernandez, M. Laine, P. Weisz and H. Wittig, Low-energy couplings of QCD from current correlators near the chiral limit JHEP 0404, 013 (2004) [arXiv:hep-lat/0402002].

[8] W. Bietenholz and S. Shcheredin, Overlap hypercube fermions in QCD with light quarks arXiv:hep-lat/0508016.

[9] S. Shcheredin and W. Bietenholz, Low energy constants from the zero mode contribution to the pseudo-scalar correlator arXiv:hep-lat/0508034.

[10] T. DeGrand and S. Schaefer, Improving meson two-point functions in lattice QCD Comput. Phys. Commun. 159, 185 (2004) [arXiv:hep-lat/0401011].

[11] W. A. Bardeen, E. Eichten and H. Thacker, Chiral Lagrangian parameters for scalar and pseudoscalar mesons Phys. Rev. D 69, 054502 (2004) [arXiv:hep-lat/0307023]. 